\title{
Reflections from the past
}

\section{Peter Whittle}

Howell Tong has carried the subject of nonlinear time series models, notably that of threshold models, so far forward that one is lost in admiration and feels him to be lost from view. However, Howell was so kind as to mention my seiche analysis (Whittle, 1954) in his review paper, which stimulates me to recall those distant days. When I do so, I realise that my train of thought at that time in some respects wandered off the line which Howell has followed. The differences in emphasis are minor, but are worth noticing.

Howell has largely considered piecewise ARMA models of general but finite order. By 'piecewise' I imply the greatly generalised threshold property: that the ARMA coefficients are themselves piecewise constant functions of the variables of the system.

My analysis (there was really no model) was concerned with seiches - oscillations of bodies of water in a confined space - but in the sea. A degree of confinement in coastal regions can produce identifiable seiches, damped by propagation of energy into the open sea but maintained by diffuse propagation from that source. No finite-dimensional model could fully reproduce such a situation; oscillations tend to be purer in frequency than a finite-dimensional autoregression could produce.

The 1954 paper considered the situation of a rocky channel opening on to the sea with an island placed at some distance from its mouth. A smoothed periodogram of the data (on water height at a fixed point in the channel) demonstrated several peaks. The two strongest of these could be clearly identified with two seiches in the channel, one generated by reflection between its landward end and the island and the other by reflection between its landward (closed) and seaward (open) ends. These had periods of roughly 11 and 14 units of time respectively.

Nonlinearity revealed itself in the periods of the remaining spectral peaks, which turned out to be sums of integral mutiples of these two fundamental periods. In other words, subharmonic resonance - a phenomenon which I think will be observed only if the fundamental oscillations are sufficiently pure. The explanation was that conditions at the mouth of the channel changed if water height there exceeded a certain level, and this occurred at times when the fundamental oscillations reinforced each other, implying period relationships of the type observed. So, a simple mechanism, simply diagnosed and simply explained. Incomplete as an analysis of course, because the intermittent effect of threshold-crossing must have an effect upon the fundamental wave trains as well, but this is presumably a second-order effect. I was fortunate in my data, because models of the generality which Howell considers will not often have the purity of periodic components which allows such clear effects and explanation. However, sometimes they do, and Howell arms us for the cases when they do not.

\section{Received 2 February 2011}

\author{
Peter Whittle \\ Statistical Laboratory \\ Centre for Mathematical Sciences \\ University of Cambridge \\ Cambridge, CB3 0WB \\ England \\ E-mail address: whittle@statslab.cam.ac.uk
}

